\title{
Performance Analysis for MANETs using certain realistic mobility models: NS-2
}

\author{
R. Kumari ${ }^{*}$, P. Nand ${ }^{2}$ \\ $1^{*}$ Dept. of CSE, SCSE, Galgotias University, Gr. Noida, India \\ ${ }^{2}$ Dept. of CSE, SCSE, Galgotias University, Gr. Noida, India \\ *Corresponding Author: ranichoudhary04@gmail.com, Tel.: 9971733493
}

Available online at: www.isroset.org

Received 26/Dec/2017, Revised 09/Jan/2018, Accepted 22/Feb/2018, Online 28/Feb/2018

\begin{abstract}
In order to know about how a protocol carries out when deployed in a real scenario, it is jussive that the simulation gathers the true nature of the physical scenario and the mobility of nodes, for all cases it may not be possible. For example, consider a scenario where a set of nodes are deployed in a release-rescue operation. Even though the mobility of the nodes can be gathered with certain realistic mobility models and techniques, the node doesn't gather the uneven areas, catastrophic failure of the nodes, and so on, in which the nodes operate. This paper focuses on analyzing the AODV Performance over different variants of realistic mobility models by the use of ns-2.
\end{abstract}

Keywords - Privacy, Authentication, Vehicular Networks, VANET, Intelligent Transportation System (ITS), security;

\section{INTRODUCTION}

Taking last few years into consideration, interest is on a hike in the research community for simulation study of performance in MANETs and there has been a lack of infrastructure, which is apt for MANETs to be deployed in a realistic scenario. A simulation study generates an idea of how a protocol carries out when it is practically applied. This approach is quite similar to the prototyping model in software engineering knowledge domain. However, the main problem that lies in the simulation study is revolving around the following,

1) Dynamic environment of the network topology. 2) The physical environment in which the nodes operate.

\section{The ns-2 network simulator}

NS-2 is generally used by researchers, for research in network field. Since it is an open source discrete event simulator [30].Both wired and wireless networks are supported by this simulator. It can perform simulation for many network protocols like UDP (user datagram protocol), TCP(Transmission Control Protocol) etc. Recently, for simulating the large satellite and ad-hoc wireless networks support has been added. The NS-2 simulation software was developed at the University of Berkeley. This software is carried out by active community. The latest version ns-2 2.35 is taken into consideration in this paper.

Linux is used for operating the standard ns-2 distribution. Cygwin (Linux Emulation for windows) is available to run this software. The figure 1.1 shows ns-2 operates in the Fedora Environment and the simulations performed have been run here.

\begin{tabular}{|c|}
\hline NS -2 ver. 2.35 \\
\hline Fedora \\
\hline Figure.1.1: NS-2 over Fedora
\end{tabular}

Split-programming model is also provided by NS-2, which is implemented with the use of $\mathrm{C}++$, while simulation control is done by scripting language ( $\mathrm{Tcl})$.

NS-2 is beneficial for the following

1) The approach provided by NS-2 has several benefits over programming methods.

2) It can create animation file or a detailed trace file for every ad-hoc network.

3) Helpful in analyzing routing behavior.

\subsection{Wireless Support}

NS-2 supports the simulation for the multi-hop wireless network. This simulation covers the LL and the physical layer of the OSI model. NS-2 provides in-built various routing protocols such as DSR ,DSDV, etc. It also provides the traffic and the mobility control.

IEEE 802.11standard for wireless LANs is considered as Distributed Coordination Function (DCF) mode being used as MAC layer protocol for NS-2. The same characteristics are used by Lucent's Wave LAN (radio model) [30]. Wave LAN is created as a shared-media radio with nominal radio 
range and "br- bit rate" of 250 meters and $2 \mathrm{Mb} / \mathrm{s}$ respectively. A free space propagation model and two-ray ground reflection model combines to make signal propagation model.

\subsection{Other add-on utilities}

If we say about added utilities, a standard ns-2 distribution can be used to design the movement of the nodes, specifying the traffic flow among nodes or to the extent of visualizing the network topology and traffic flow as an animation. Some of these utilities which are used in the experiments are described below-

(i) cbrgen.tcl: The NS package contains a traffic generator utility existing in the folder $\sim$ ns/indep-utils/cmu-scen-gen/ (where $\sim \mathrm{ns}$ is ns-directory, for eg., for the ns-2.35 version/home/administrator/ns-allinone2/ns-2.35/). By using this utility trace file can be generated effectively. These trace files will present the duration, the type and the traffic rate which is in the network. To invoke and use this utility we call the TCL script as stated below:-

\section{\$ nscbrgen.tcl [parameters]}

Parameters:-

- Traffic Type: TCP or CBR

- Seed: random number generator picks this number to begin with.

- NR : No. of node

- NC : Maximum Number Of Connections.

- Rate: Bit rate ( No. Of Packets/Sec)

The > directive is generally opted for presenting (writing) the output values to a particular file. And hence we can use this file for giving input to the TCL script.

(ii)SETDEST utility: Secondly a SETDEST utility is also available as an add-on utility, the same was developed at CMU, and is basically opted to generate node motions according to the known Random Waypoint Model [31] the SETDEST Utility is generally available under $\sim$ ns/indeputils/cmu-scen-gen/setdest directory and it consists of Makefile and setdest $\{. \mathrm{cc}, . \mathrm{h}\}$. The utility correctly runs by using the following arguments:-

\section{./setdest [-n num_of_nodes] [-p pausetime] [-s maxspeed] [-t simtime] $\backslash[-\mathrm{x} \operatorname{maxx}][-\mathrm{y} \operatorname{maxy}]>[$ outdir/movement- file]}

To show the output file following file does the job outdir/movement file and it is added with the simulation script itself.

(iii) Network Animator (NAM): To be brief on what "NAM" stands for is, it is basically the Network Animator which acts as a graphical tool and is used to trace the flow of data packets in a network and to visualize the simulation results graphically as shown -

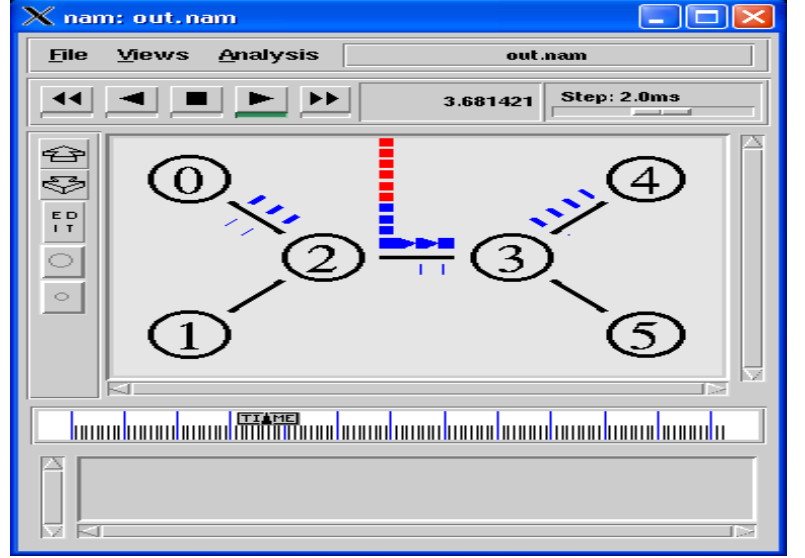

Figure 1.2: Screenshot of NAM Output Window

\subsection{Advantages and Disadvantages of ns-2}

Advantages highlight the necessity of a software tool be it NS-2 or others. The most proper advantages do provide benefits at a large extent, the NS-2 simulator is an opensource software and thereby it is freely available. Further, it is easily extensible and any changes like addition or modification of routing protocols are comparatively easy. However, the complementary opposite of the former, is the complex coding - Basically it requires understanding of two basic languages i.e $\mathrm{C}++$ and TCL .These if judged on the need are very much needed to develop any new protocol because of the split-programming approach. Besides all these pros and cons, the user interface is shabby and the learning curve does have a sharp inclination, indeed not gradual.

\subsection{Mobility Modeling}

Several mobility models are opted just to simulate the motion of nodes in an ad hoc network [31]. The mobility model hence chosen should correctly project the motion of the nodes because usually the mobility of the nodes affects their performance. The mobility models are classified into two variants, on the basis of temporal and spatial dependency between the nodes in the network -

1. $\operatorname{GMM(Group~Mobility~Models)~}$

2. $\operatorname{EMM(Entity~Mobility~Models)~}$

The below sections give the detailed description on the mobility models -

\subsubsection{Entity mobility models}

The entity mobility models which is abbreviated as EMM, describes the motion of nodes in which movement of a particular node is independent of movement of other nodes. Variety of such mobility models have been proposed till date. This section provide a brief idea on a few of those models used for the research.

i. RWM (Random Waypoint Mobility Model) :

The random waypoint mobility model (RWM) describes the random movement of nodes in an area. The movement pattern of a node in this model is as described below -: 
1) The node stays in particular location for a period of time, is known as 'pause time'.

2) Once this time slot expires, a random destination is chosen by the node in the simulation terrain and a velocity which is evenly distributed between 'minspeed' and 'maxspeed'.

3)Then the node propagates towards a new destination chosen by it with an even speed.

4) On arrival to the destination, it again becomes stationary for the duration of 'pause time' and continues the process.

5) The RWM is extensively used in designing the movement of nodes for the study of protocol performance [5] [10] [11] [16].

The RWM is not efficient enough to capture the realistic movement of nodes. Further, it also fails to converge when the pause time increases and the av. speed of the nodes decreases over time [31]. The proposed solutions for this problem are given below:-

1) To assign a non-zero minimum speed to the nodes

2) To "cut off" some initial part of the simulation for the stabilization of nodes' movements.

\section{ii. Gaussian Markov Mobility Model(GMM):}

This model was used initially to simulate the motion of nodes in Personal Communication System (PCS) [32]. In Gauss Markov Mobility model, we can use a tuning parameter to change the level of random motion of nods. Initially, each and every mobile node has direction and current speed individually. After a fixed interval of time n, the direction and speed of every node is updated based upon the $(n-1)^{\text {st }}$ instance using these following equations -

$$
\begin{aligned}
& P_{n}=\alpha_{P n-1}+(1-\alpha) \bar{P}+\sqrt{\left(1-\alpha^{2}\right)} P_{x_{n-1}} \\
& Q_{n}=\alpha_{Q n-1}+(1-\alpha) \bar{Q}+\sqrt{\left(1-\alpha^{2}\right)} Q_{x_{n-1}}
\end{aligned}
$$

Where,

- $Q_{n}$ and $\mathrm{P}_{n}$ are variables for direction and the new speed the Mobile Node at time interval $n$;

- $\alpha$, where $0 \leq \alpha \leq 1$, is the tuning variable used to differ the randomness;

- $Q$ and Pare constants indicating the mean value of direction and speed as $n \rightarrow \alpha$; and $\boldsymbol{P}_{x_{n-1}}$ and $Q_{x_{n-1}}$ are random parameters from a Gaussian distribution

\section{iii. City Section Mobility (CSM)Model}

This mobility model depicts the real motion of vehicular traffic in a part of the city since it stops the motion of the nodes.

The City Section Mobility (CSM) Model visualizes the motion of mobile nodes in a city street. For example, it can showcase the movement of cars on the streets. The streets and speed cut offs are generally dependent on the type of city simulated. For example, in the Manhattan grid mobility model [31], movement of the nodes is modeled based on the Manhattan city streets. In CSM mobility model, the entire simulation area is partitioned into several grids representing streets in a city. Initially, these mobile nodes are spread out randomly in that simulation area. They select a random destination and opt for the path that yields minimum cost. On arrival to the destination, it stops for an interval and proceeds further accordingly.

\subsubsection{Group mobility(GM) models}

Group Mobility Model showcases independent movement of the nodes in a MANET. However, there are environments in which the nodes basically lead to the formation which quite lies in a category of "clusters", and based on the motion of other nodes, the nodes propagate within the clusters. For eg. Just consider the battlefield environment where groups of soldiers are forwarded in a battlefield. In this case every soldier's movement in the group is dependent upon the Commander of the group. The group mobility models capture such motion of nodes. This section removes dust from, the Reference Point Group Mobility model and is explained accordingly, It is basically a group mobility model.

\section{$i$. The RPGM (Reference Point Group Mobility Model)}

The RPGM model presents the motion of the particular individual nodes and the random motion of a group of nodes Intra group. An individual node in a group moves randomly depending upon the direction and velocity of its "logical center", which is updated at predefined time intervals. Group motion vector is calculated with the help of the logical center of a group. The group motion vector completely characterizes the motions of the nodes within the group. Further, each node also selects is direction and velocity according to a random motion vector and a fixed reference point. The random motion of both the logical center and the individual nodes are calculated using the Random Waypoint Mobility (RWM) Model.

The RPGM model can be used to depict several scenarios of deployment such as movement of soldiers in a battlefield, avalanche rescue, etc.

\subsection{Performance Analysis of MANET Routing Protocols}

The existing research covers the analysis of routing protocol by using the random waypoint model. In this model the nodes are placed at the random position. This work will evaluate the work on the real world scenario. The real world scenario analysis will enable us to determine the optimized performance of the routing protocol in the real world [5][10][11]. The next section contains the performance evaluation of the AODV under real world scenario.

\subsubsection{Performance evaluation of AODV}

The AODV is a On-demand routing protocol that generates the routing table on demand. The performance of the protocol is better than the other table driven routing protocol 
in terms of the PDR and the latency. It is because of the availability time of routing table. This protocol adapts itself with node mobility and link changes. The performance evaluation of the AODV over defined network scenario with PDR, routing load and the latency as parameter is discussed in next section.

\subsubsection{Experimental Setup and Metrics}

The ns-2 simulator is used for the simulation. This section covers the description of scenario and the metrics. It also covers traffic pattern for the simulation.

\section{(i) The traffic pattern}

"cbrgen.tcl" script was used for generating the file of traffic pattern. The following parameters were used:-

Table 1.1: Traffic pattern

\begin{tabular}{|c|l|}
\hline Packet Rate & $5 \mathrm{pkts} / \mathrm{sec}$ \\
\hline Packet Size & 512 bytes \\
\hline Type of traffic & Constant Bit Rate \\
\hline Max. no. of connections & 22 \\
\hline
\end{tabular}

\section{(ii) Scenario description}

Bonn Motion software was used for generating the scenario. It is a Java-based software. It can also create and analyze the mobility scenario of nodes. In an ad-hoc network, it generates a trace file for the motion of nodes. This file can be imported into NS-2. Some mobility models like Gauss Markov mobility model, the Reference Point Group mobility model, Random Waypoint model are supported by Bonn Motion software. The real world scenario is depicting by following metrics:-

\begin{tabular}{|l|l|}
\hline \multicolumn{1}{|c|}{ Characteristics } & \multicolumn{1}{|c|}{ Value } \\
\hline Dimensions & $1000 * 1000$ \\
\hline Mobility Model & $\begin{array}{l}\text { Reference Point Group Mobility Model } \\
\text { RPGM }\end{array}$ \\
\hline $\begin{array}{l}\text { Avg no. of nodes in a } \\
\text { group }\end{array}$ & 12 \\
\hline Min. speed & $1 \mathrm{~m} / \mathrm{s}$ \\
\hline Max. speed group & $6 \mathrm{~m} / \mathrm{s}$ \\
\hline No. of nodes & 55 \\
\hline $\begin{array}{l}\text { Probability of } \\
\text { change }\end{array}$ & $60 \mathrm{sec}$ \\
\hline Pause time & \\
\hline
\end{tabular}

Table 1.2: Parameters for the real world scenario

\section{(iii) Metrics:}

These metrics were used for analyze the performance-

a) Packet Delivery Fraction: It is the ratio of the total number of received packets to the total number of sent packets by the nodes in the process of simulation.

$$
P D F=\frac{R p}{S p}
$$

Where, Rp and Spare the parameters used for total number of received packets and total number of sent packets respectively.

Successful delivery of packets to the application layer by the protocol can be estimated by PDF. If the value of PDF is high, then it shows that mostly packets are received by higher layers.

b) Normalized Routing Load (NRL): It is the ratio of the total number of packets successfully transmitted to the total number of packets successfully received.

$$
N R L=\frac{S p}{R p}
$$

Where, $\mathrm{Rp}$ and $\mathrm{Sp}$ are the parameters used for total number of received packets and total number of sent packets respectively.

This estimate gives an idea about the efficiency of routing protocol. It also defines that how many routing packets it can send per data packet and how efficiently it can update the routing information. As the value of NRL increase, it decreases the capability of the protocols and increases the overhead of routing packets.

c) Latency: It can be defined as the average delay in transmitting packets between the two nodes and can be calculated as under.-

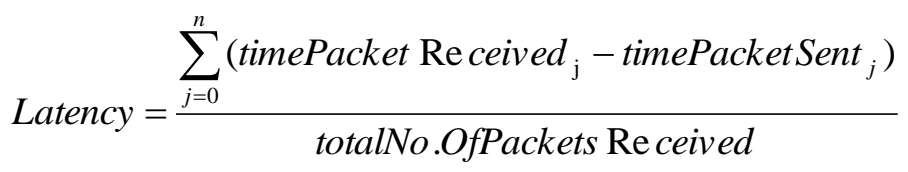

Increased value of Latency shows the congested network and due to that the routing protocol cannot perform well. The values of Latency can be calculated by the application.

\section{(iv) Research methodology}

There are three different parameters in the real world scenario which are as follows:-

1) Pause time,

2) The total no. of nodes and

3) Avg. no. of nodes in a group

\subsubsection{Result}

i. Effect of changes in the no. of nodes

The effect on Packet Delivery Fraction, Normalized Routing Load and Latency was studied on the variation of nodes from 50 to 100 .The results are shown in table 1.3 and fig. $1.3,1.4$ and 1.5 . 


\begin{tabular}{|c|c|c|c|}
\hline $\begin{array}{c}\text { Number } \\
\text { of } \\
\text { Nodes }\end{array}$ & Latency (sec) & $\begin{array}{c}\text { Packet } \\
\text { Delivery } \\
\text { Fraction (\%) }\end{array}$ & $\begin{array}{c}\text { Normalized } \\
\text { Routing } \\
\text { Load }\end{array}$ \\
\hline 50 & 0.016738278 & 99.92438 & 0.2670694 \\
\hline 60 & 0.016566893 & 100 & 0.3188803 \\
\hline 70 & 0.014576984 & 100 & 0.42268674 \\
\hline 80 & 0.033688957 & 99.96756 & 0.47658385 \\
\hline 90 & 0.020179137 & 99.96761 & 0.49718322 \\
\hline 100 & 0.020737591 & 99.852444 & 0.556427 \\
\hline
\end{tabular}

Table 1.3: Effect of changes in the no. of nodes

As the no. of nodes in the network increases the packet delivery fraction (PDF) decreases. This happens due to the congestion in network increase as number of nodes increase. So, that it increase retransmission in the number of lost packets.

Since AODV use table driven approach, result in increase the size of the network along with the delay in the processing at the nodes. Increase in the routing packets results in to increasing the no. of nodes and hence, increasing the Normalized Routing Load (NRL).

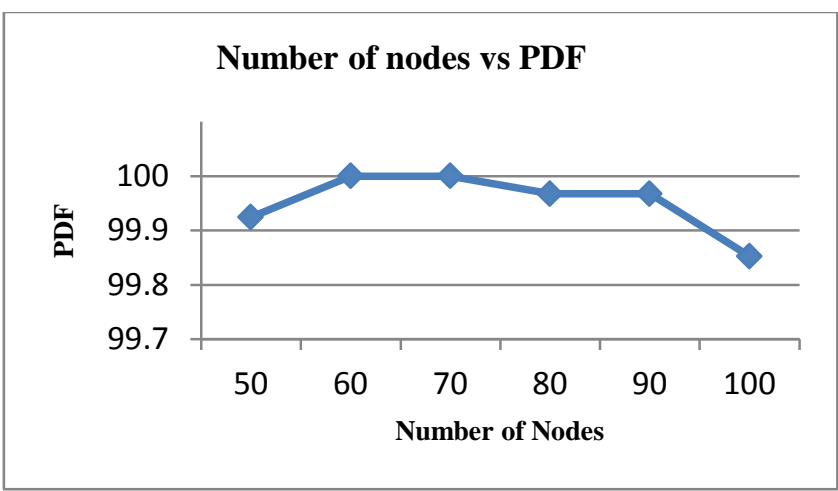

Fig. 1.3: PDF vs Number of nodes



Fig. 1.4: Latency vs number of nodes

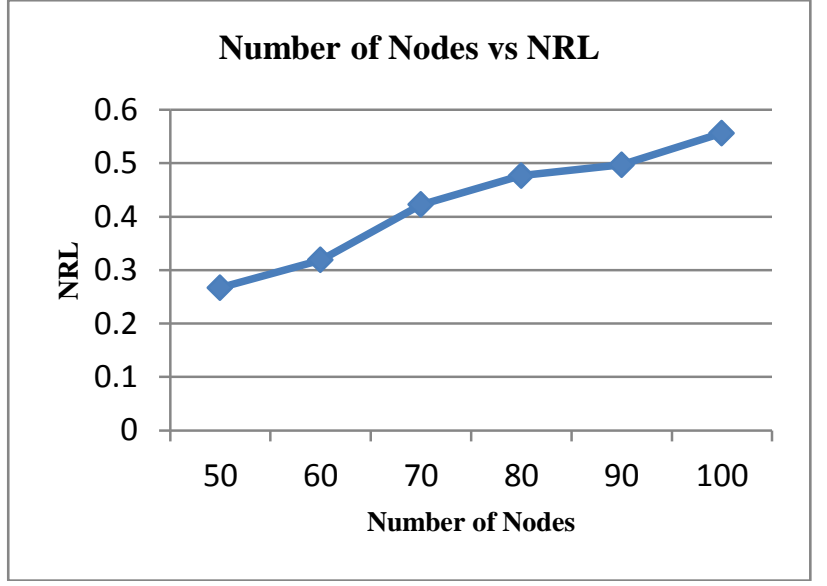

Fig. 1.5: Routing Load Vs Number of nodes

The highest value of PDF is 100 at the $60 \& 70$ node scenario. The lowest value of Latency is .014at the 70 node and the lowest value of NRL is 026 at the node 50 as shown in fig.1.3, 1.4 and 1.5.

\section{i. Effect of different values of the pause time}

The effect of different values of the pause time on all the three metrics are shown by table 1.4 and the corresponding graphs are shown in figures 1.6, 1.7 and 1.8. Increased value of Packet delivery Fraction can be estimated by the variation in Pause time. Increased pause time results in to decreased congestion in the network and the relative mobility of the nodes also decreases.

\begin{tabular}{|c|c|c|c|}
\hline $\begin{array}{c}\text { Pause } \\
\text { Time } \\
(\mathbf{s e c})\end{array}$ & $\begin{array}{c}\text { Latency } \\
\text { (sec) }\end{array}$ & $\begin{array}{c}\text { Packet } \\
\text { Delivery } \\
\text { Fraction } \\
\text { (\%) }\end{array}$ & $\begin{array}{c}\text { Normalized } \\
\text { routing } \\
\text { load }\end{array}$ \\
\hline 10 & 0.007634372 & 99.87318 & 0.25598268 \\
\hline 20 & 0.007683255 & 99.956466 & 0.25532915 \\
\hline 30 & 0.007524965 & 99.91636 & 0.25413962 \\
\hline 40 & 0.011312819 & 100 & 0.27755056 \\
\hline 50 & 0.011314601 & 100 & 0.2742716 \\
\hline
\end{tabular}

Table 1.4: Effect of different values of the pause time

The latency decreases with the increase in the pause time. The change in the pause time doesn't make difference to the network topology that results in the same routes in the routing tables. It results in the less time in route discovery and maintenance. This will reduces the number of routing packets in the network, thereby decreasing the NRL. 




Fig. 1.6: PDF vs Pause time

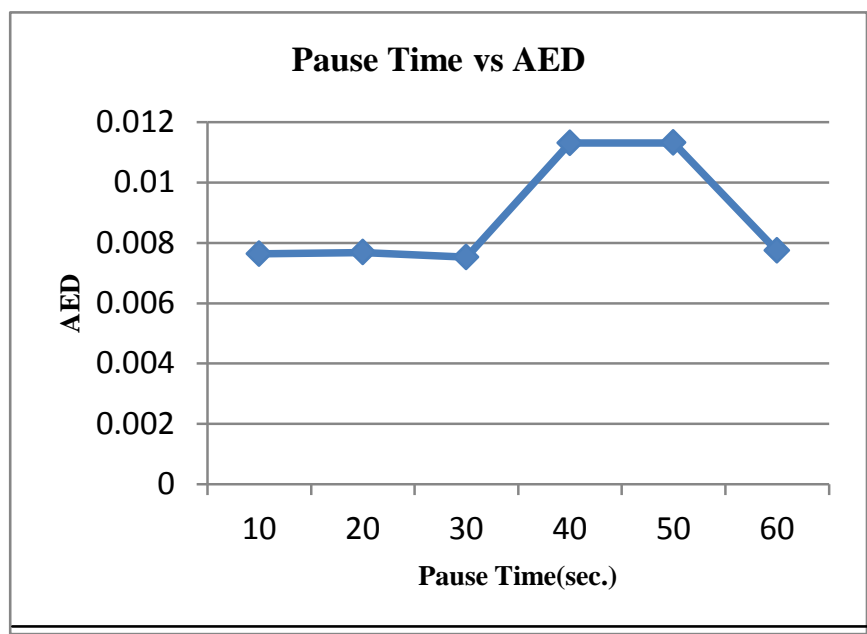

Fig. 1.7: Pause time vs Latency

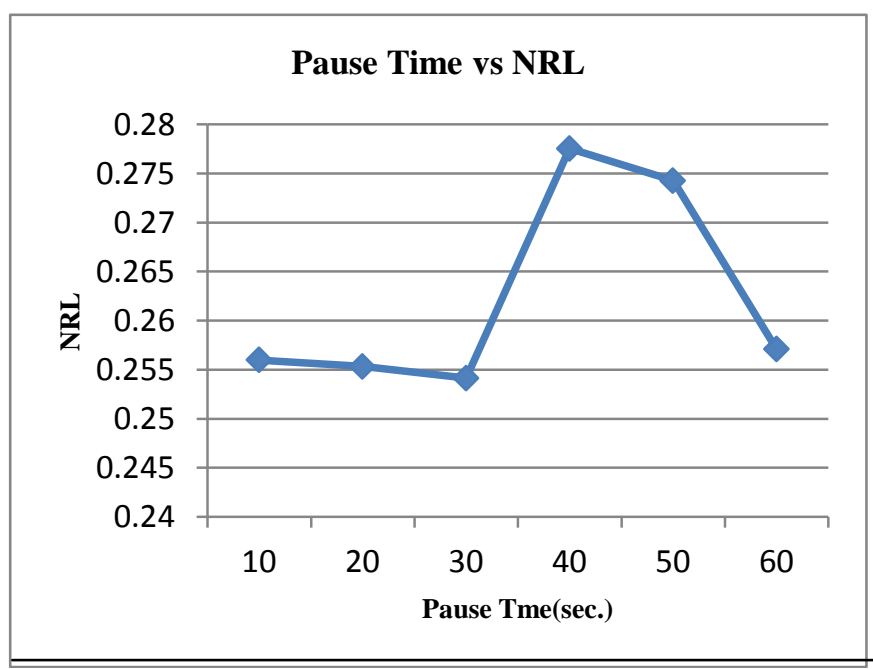

Fig. 1.8: Pause time vs NRL
It can be analyzed that we obtain the highest value of PDF at pause time of $40 \mathrm{sec}$. and $50 \mathrm{sec}$., the lowest value of NRL at pause time of $30 \mathrm{sec}$. and the lowest value of latency at 30 sec .

i. Analysis of scenario by changing the average number of nodes

The effect of changing the average no. of nodes on the three different metrics is shown in table 1.5.

\begin{tabular}{|c|c|c|c|}
\hline $\begin{array}{c}\text { Average } \\
\text { Number } \\
\text { of } \\
\text { Nodes }\end{array}$ & Latency (sec) & $\begin{array}{c}\text { Packet } \\
\text { Delivery } \\
\text { Fraction } \\
(\%)\end{array}$ & $\begin{array}{c}\text { Normalized } \\
\text { routing load }\end{array}$ \\
\hline 5 & 0.011453271 & 100 & 0.27755056 \\
\hline 6 & 0.015189819 & 99.95736 & 0.2992832 \\
\hline 7 & 0.006558823 & 99.91546 & 0.25477807 \\
\hline 8 & 0.006717324 & 100 & 0.25575547 \\
\hline 9 & 0.018597672 & 99.87298 & 0.3182111 \\
\hline 10 & 0.006748278 & 99.91448 & 0.2570794 \\
\hline
\end{tabular}

Table 1.5: Effect of changing the average number of nodes

The graphs 1.7, 1.8 and 1.9 represent the three metrics.

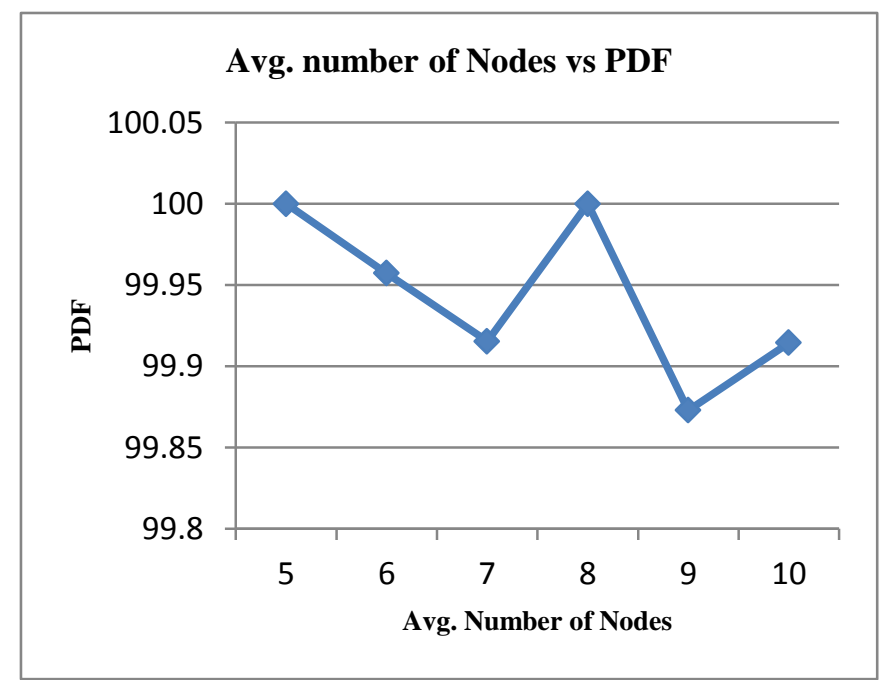

Fig.:- 1.9: Average no. of nodes vs PDF

From figure 1.9 the increase in the average number of nodes increases the PDF. It is because with the increase in the average number of nodes, the density increases resulting congestion in the network. This results in the decrease in the PDF. 
Figure 1.10 shows the effect of increasing the average no. of nodes on the latency. It is analyzed that the density increases with the latency decreases. It also noticed that the lower latency achieved by not using the source routing.

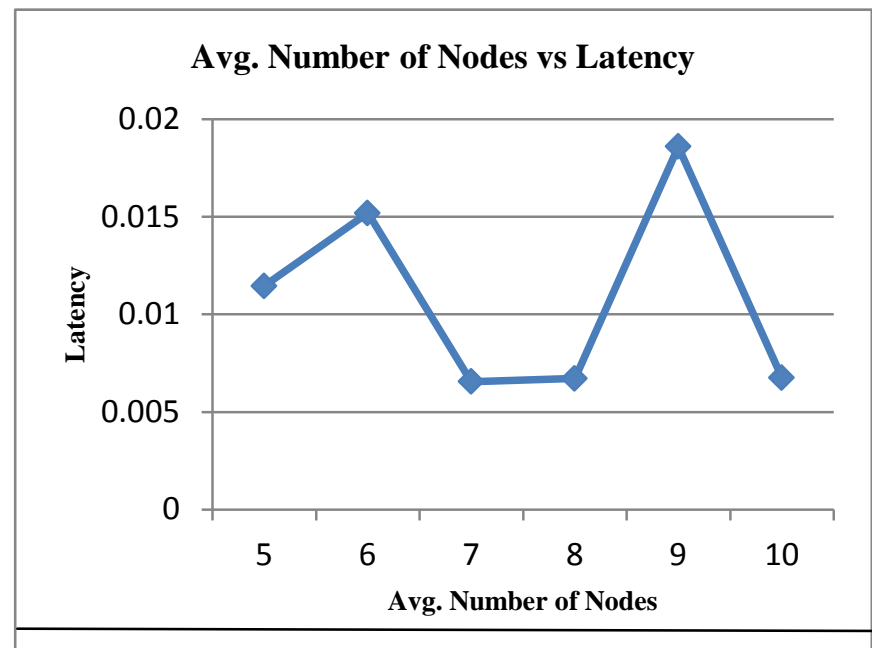

Fig. 1.10: Average no. of nodes vs Latency

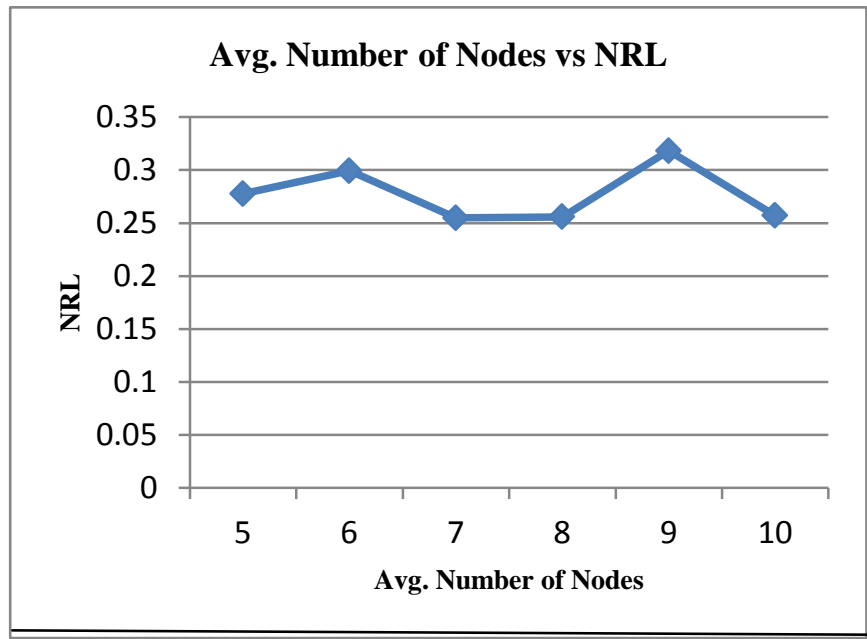

Fig. 1.11: Average no. of nodes vs NRL

Figure 1.11 shows that the AODV has less routing overhead for achieving a highest load of 0.32 , when there is 9 nodes in a group.

\subsection{Conclusion}

This paper covers the performance analysis of the AODV routing protocol in the real world scenario by varying the pause time and the number of nodes. The analyzed parameters are PDF, latency and the normalized routing load. The higher value of PDF and lower values of the latency and NRL shows the optimized performance of the AODV routing protocol in the real world scenario. In future this protocol can be extended to improve the security so that it can handle various attacks.

\subsection{Summary}

It gives a simulation centric approach for performance study of routing in MANETs. It yields and thereby provides an introduction to the NS-2 simulator and its added facilities. It is also inclined towards some simulation-based apt added utilities for an ad hoc networking research. To bind each and every bit of NS-2 simulator, some of its advantages and the disadvantages are also described.

We have also discussed about some mobility models used for simulating the motion of nodes in an ad hoc network. Several other mobility models are being developed which try to form an alias or mimic the scenario in which the nodes are released. These kinds of models are very useful in attaining a broader visualization of the performance of different routing protocols in realistic deployments.

\section{REFERENCES}

[1] C.S. Murthy, B.S. Manoj, "Ad Hoc Wireless Networks :Architectures and Protocols", Prentice Hall Publishers, May 2004, ISBN 013147023X.

[2] C.K. Toh, "Ad Hoc Mobile Wireless Networks: Protocols and Systems", Prentice Hall publishers, December 2001, ISBN 0130078174.

[3] C. Perkins and P. Bhagwat, "Highly Dynamic DestinationSequenced Distance-Vector Routing (DSDV) for Mobile Computers". In Proc. of the ACM SIGCOMM, October 1994.

[4] S. Murthy, J.J. Garcia-Luna-Aveces, "A Routing Protocol for Packet Radio Networks, " Proc. ACM International Conference on Mobile Computing and Networking, pp. 86-95, November, 1995.

[5] C.C. Chiang, "Routing in Clustered Multihop, Mobile Wireless Networks withFading Channel," Proc. IEEE SICON '97, Apr. 1997, pp. 197-211.

[6] S R. Das, C E. Perkins, E M. Royer and M K. Marina ."Performance Comparison of Two On-demand Routing Protocols for Ad hoc Networks. "IEEE Personal Communications Magazine special issue on Ad hoc Networking, p. 16-28, February 2001.

[7] Z.J Haas, "A new routing protocol for the reconfigurable wireless network". In Proceedings of the 1997 IEEE 6th International Conference on Universal Personal Communications, ICUPC '97, San Diego, CA, pp. 562 - 566,October 1997;

[8] K.Sanzgiri, B. Dahill, B. N. Levine, C. Shields and E M. B royer. "A Secure Routing Protocol for Ad Hoc Networks" (ARAN) In International Conference on Network Protocols (ICNP), Paris, France, November 2002.

[9] S Basagni, M Conti, S Giordano, I Stojmenovic, "Mobile Ad Hoc Networking”, ISBN: 0-471-37313-3, Wiley-IEEE Press: Chapter 12: Ad hoc networks SecurityPietroMichiardi, RefikMolva.

[10] Y Hu, D B. Johnson, A Perrig. "SEAD: Secure Efficient Distance Vector Routing for Mobile Wireless Ad Hoc Networks", Fourth IEEE Workshop on Mobile Computing Systems and Applications (WMCSA '02), pp: 3-13, Jun 2002.

[11] Y Hu, A Perrig, D B. Johnson. "Ariadne: A secure On-Demand Routing Protocol for Ad hoc Networks"MobiCom 2002, Atlanta, Georgia, USA, September 23-28, 2002. 
[12] A. Perrig, R. Canetti, D. Tygar, and D. Song, "The TESLA Broadcast Authentication Protocol," Cryptobytes,, Volume 5, No. 2 (RSA Laboratories, Summer/Fall 2002), pp. 2-13.

[13] P. Papadimitratos and Z. Haas. "Secure routing for mobile ad hoc networks" (SRP) SCS Communication Networks and Distributed Systems Modeling and Simulation Conference, pp. 27--31, January 2002.

[14] S. Capkun, L. Buttyan and J-P Hubaux. "Self-Organized PublicKey Management for Mobile Ad Hoc Networks ", IEEE Transactions on Mobile Computing, Vol. 2, No. 1, pp. 52-64,JanMar 2003.

[15] W Wang, Y Zhu, B Li. "Self-Managed Heterogeneous Certification in Mobile Ad Hoc Networks ", in the Proceedings of IEEE Vehicular Technology Conference (VTC 2003), Orlando, Florida, 10/6-9, 2003.

[16] J. Kong, P. Zerfos, H. Luo, S. Lu, and L. Zhang. "Providing robust and Ubiquitous Security support for Mobile Ad Hoc Networks ", Proceedings of the 9th International conference on Network Protocols (ICNP), Riverside, California, USA, November 11-14, 2001.

[17] E C. H. Ngai and M R. Lyu. "Trust- and Clustering-Based Authentication Services in Mobile Ad Hoc Networks", 24th International Conference on Distributed Computing Systems Workshops - W4: MDC (ICDCSW'04), Hachioji, Tokyo, Japan, 3/23-24, 2004.

[18] L. Zhou and Z. Haas. "Securing Ad Hoc Networks", IEEE Network magazine, special issue on networking security, Vol. 13, No. 6, November/December 1999.

[19] M C Morogan "Certificate Management in Ad Hoc Networks", Symposium on Applications and the Internet Workshops (SAINT'03 Workshops), pp. 337,January 27 - 31, 2003.

[20] T. Camp, J. Boleng, and V. Davies. "A Survey of Mobility Models for Ad Hoc Network Research",in Wireless Communication \& Mobile Computing (WCMC): Special issue on Mobile Ad Hoc Networking: Research, Trends and Applications, vol. 2, no. 5, 2002.

[21] B Liang, Z. J. Haas, "Predictive distance-based mobility management for multidimensional PCS networks", IEEE/ACM Transactions on Networking (TON), Volume 11, Issue 5 , Pages: $718-732$, October 2003

[22] J. Yoon, M. Liu, and B. Noble."Random waypoint considered harmful," in Proc. of IEEE INFOCOM '03, vol. 2, pp. 13121321, March 2003.

[23] M G Zapata, "SecureAdhoc On-Demand Distance Vector Routing”, ACM Mobile Computing and Communications Review (MC2R), pp:106--107, July 2002.

[24] R. Kumari, P. Nand, "Performance Analysis of existing Routing Protocols" International Journal of Scientific Research in Computer Science and Engineering (IJSRCSE), ISSN-2320-7639, Vol. 5 No. 5, October-2017.

\section{Authors Profile}

Ms. $R$ Kumari pursed Bachelor of Technology from UPTU of Lucknow,in 2008 and Master of Technology from IET in year 2010.She is Gold Medalist in M.tech. She is currently pursuing Ph.D. and currently working as Assistant Professor in Department of Computer Science, Chitkara University, Chandigarh. She is a member of ACM since 2016. She has published more than 15 research papers in reputed international journals including Thomson Reuters and conferences including IEEE and it's also available online. Her main research work focuses on Software Engineering, Network Security, Data Structure, Big Data Analytics, Data Mining, IoT and Wireless Sensor Network. She has 8 years of teaching experience and 2 years of Research Experience.

Dr Parma Nand is Dean, Computing Science \& Engineering, Galgotias University, Greater Noida. He has over 24 years of teaching and research experience. He has expertise in Wireless and Sensor Network, Cryptography, Algorithm and Computer Graphics. He has been Ex-President of National Engineers Organization. $\mathrm{He}$ is senior member of IEEE (USA). He is member


Executive Council of IEEE UP section, member Executive Committee IEEE Computer and Signal Processing Society, member Executive of India council Computer Society, member Executive Council Computer Society of India, Noida section and has acted as an observer in many IEEE conferences. He is also having active memberships of ACM, CSI, ACEEE, ISOC, IAENG, and IASCIT. $\mathrm{He}$ is life time member of Soft Computing Research Society (SCRS) and ISTE. He has published more than 60 papers in peer reviewed international/national journals and conferences. He is an active member of advisory/technical program committee of reputed International/National conferences \& reviewer of number of reputed Journals e.g. Springer, Elsevier Journal Computers \& Electrical Engineering. 